\title{
Input modality and the serial position curve in short-term memory
}

\author{
R. CONRAD AND A. J. HULL \\ APPLIED PSYCHOLOGY RESEARCH UNIT, CAMBRIDGE, ENGLAND
}

\begin{abstract}
Results are presented which confirm data already published by Corballis (1966), and Murray, (1966). These show that when a sequence of letters or digits is presented for immediate recall, there is a marked difference in the serial position curve of errors according to whether presentation is auditory or visual. This difference specifically shows as a virtual absence of recency in the visual presentation condition.
\end{abstract}

When, without thinking too much about it, we think of the serial position (SP) curve for retention of a learned sequence of items, what generally comes to mind is an error curve high in the middle and down at the ends. This is true for rote learning of long lists (McCrary \& Hunter, 1953), or for immediate recall of short lists (Crowder \& Melton, 1965). It is the latter only that concerns us here. Although the general shape of this curve can be distorted by violent changes in the presentation or recall conditions, such as von Restorff noted or Posner (1964) has reported, these are always accepted as distortions of a basic shape.

Whenever classical SP curve data have been published, test material has always been presented auditorily. Whenever (more rarely) material has been visually presented (Conrad, 1965), a marked decline of recency is shown-the last item presented is almost the worst recalled. Two recent experiments, however (Corballis, 1966; Murray, 1966), specifically compare the SP curves when presentation is either visual or auditory. In both studies the SP curves are different, but only for the later positions. Neither sets of data are entirely satisfactory.

Murray frankly says (p. 15): "This was a finding which had not been predicted." The SP curves he presents showing a difference between auditory and visual presentation are composite curves pooling several presentation rates. Elsewhere in the article Murray reports a near-significant rate by input-mode interaction. His evidence on the current question therefore is not entirely noise-free. But in the present context, a particular virtue of Murray's data is that the procedure was such that the only difference between visual and auditory presentation was that in the latter the same Ss read out aloud what in the former they read silently.

In the Corballis study this was not so. In the auditory condition digit lists were tape-recorded and played back to high school students; the visual condition used an optical projection system in a separate experiment using college students. Corballis' study was further complicated. His main concern was to examine the effect of systematically varying the interval between successive digits within a single list, and he has no "conventional" condition of fixed-rate presentation. Clearly, since his visual and auditory SP curves derive from two separate experiments, Corballis, like Murray, did not expect the effect he found. Fortunately, both authors did present their SP curves. If this effect is a genuine phenomenon of behavior, it could hardly fail to be relevant to the many attempts which have been made to account for the strange but consistent shape of the SP curve. The present experiment, in fact, does no more than simply confirm the effect in a straightforward test.

\section{Method}

Two blocks, each of 10 seven-digit sequences, preceded by some practice sequences, were prepared and presented to Ss using a punched-tape programmed Bina-View display. Ss were $20 \mathrm{Naval}$ ratings individually tested. The digits were presented for a duration $100 \mathrm{msec}$ followed by an "off" period of $400 \mathrm{msec}-$ that is, two digits/sec rate. (It is worth reporting parenthetically, that a preliminary experiment showed no difference in recall when the on-off ratio using a Bina-View was varied from $50 / 450 \mathrm{msec}$ to $450 / 50$ msec). Report was written into a seven-cell answer blank and instructions emphasized guessing when necessary and writing from left to right-this instruction being monitored. For the visual presentation condition $S$ read the digits silently. In the auditory condition he read them aloud. The order of the two blocks of sequences and two test conditions were counterbalanced; each $S$ did both conditions, half starting with visual, and each $S$ had a different block for each condition.

\section{Results and Discussion}

Figure 1 shows the two resulting SP curves in terms of number of wrong digits out of 200 at each position. The general trend of the difference between visual and auditory presentation is clear enough and confirms the difference reported by Corballis (1966) and by Murray (1966). If one assumes that errors at different positions are independent of each other, simple difference tests can be made. There is obviously no difference at the first four positions. Though the difference progressively increases over Positions 5, 6, 7, significance is not reached until the last position, when the difference is massive $(p<.001)$.

In describing the experimental conditions as visual and auditory presentation, important questions are begged. The auditory SP curve shown here is essentially 


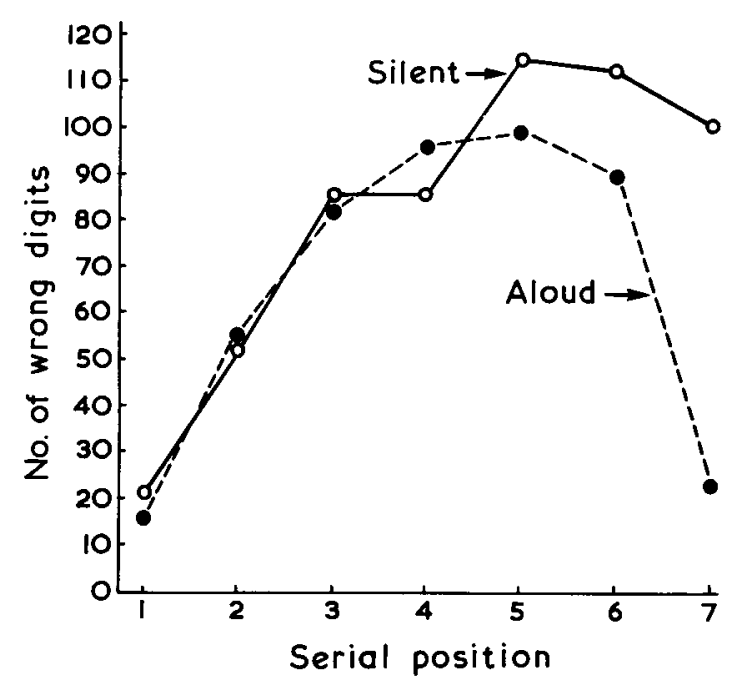

Fig. 1. Effect of input mode on serial position of errors: silent $=$ visual, aloud $=$ auditory

identical to those reported when $E$ speaks the item rather than $\mathbf{S}$ himself, and the method is free from intelligibility problems. What the nature of the input is in the visual presentation condition is unclear. But the nature of the error observed (Conrad, 1964) indicates that the input undergoes drastic recoding; the last items in the visual condition are not merely held in a short-lived visual store. Nor will any simple trace-strength model suffice which depends on input mode to determine strength, since for most of the curve there is no difference. Finally (for the time being) models of the Lepley Hull type might also have difficulty in explaining how the balance of intra-serial interference becomes shifted when sensory mode of presentation is changed. It seems wisest at present to leave these confirmatory data with minimal comment. The fact of the dramatic absence of recency when material is silently read will need to be accommodated in any model for short-term memory.

References

CONRAD, R. Acoustic confusions in immediate memory. Brit. $J$. Psychol., 1964, 55, 75-84.

CONRAD, R. Order error in immediate recall of sequences. J. verb. Learn. verb. Behav., 1965, 4, 161-169.

CORBALLIS, M. C. Rehearsal and decay in immediate recall of visually and auditorily presented items. Canad. J. Psychol., 1966, 20, 43-51.

CROWDER, R. G., \& MELTON, A. W. The Ranschburg Phenomenon: Failures of immediate recall correlated with repetition of elements within a stimulus. Psychon. Sci., 1965, 295-296.

MoCRARY, J. W. JR., \& HUNTER, W. S. Serial position curves in verbal learning. Science, 1953, 117, 131-134.

MURRAY, D. J. Vocalization-at-presentation and immediate recall, with varying recall methods. Quart. J. exp. Psychol., 1966, 18, 9-18.

POSNER, M. I. Rate of presentation and order of recall in immediate memory. Brit. J. Psychol, 1964, 55, 303-306.

Serial Isolation by Shock: Reply by David C. Raskin and Edwin W. Rubel Michigan State University

The Lippmans have offered some interesting alternative interpretations of the data we reported in this journal (Raskin, Hattle, \& Rubel, 1967), and the points which they raised require some comment. First, their speculation that electric shock isolation produced a decrease in intrusions of the isolated item can be checked empirically. We did not collect the pertinent data, and we would be pleased to see this question settled on the basis of data rather than intuition.

Second, their speculation that the shock served to bisect the list in the Shock Isolation (SI) condition raises the question as to why the usual color isolation procedure has not produced similar bisections and resultant facilitation. The verbal reports of five Ss in the SI condition did indicate that the shock served to bisect the list. This is not inconsistent with our interpretation based upon evoked orienting reflexes (OR). Perhaps our explanation was not completely understood. It was not the generalized arousal produced by shock which was responsible for the overall facilitation observed in the SI condition. As we pointed out, if increased arousal were the only important factor, then the Control Shock condition should have been equally effective. Obviously, it was not. The crucial aspect of the procedure was the consistent occurrence of the shock with the appearance of a particular serial item. We suggested that this procedure produced increased signal value, enhanced ORs, and faster learning of the shocked item. This produced ORs to items in the middle of the list much sooner than ORs normally occur without an isolating stimulus (Thompson \& Obrist, 1964). Wishner, Shipley, and Hurvich (1957) offered a similar interpretation of their findings. In essence, our procedure enhanced isolation effects because electric shock is a more salient isolating stimulus. If the shock did not consistently occur in the middle of the list (the Lippmans suggest it should not), facilitation would not be expected to occur.

\section{References}

RASKIN, D. C., HATTLE, M., \& RUBEL, E. W. The effects of electric shock isolation in serial learning. Psychon. Sci, 1967, 8, 413-414. WISHNER, J., SHIPLEY, T. E., \& HURVICH, M. A. The serial-position curve as a function of organization. Amer. J. Psychol., 1957, 70, 258-262. 